\title{
High risk of traffic crashes in pregnancy: Are there any explanations?
}

\author{
Stephen J. McCall BSc (Hons), Sohinee Bhattacharya PhD MBBS
}

See related research on page 742 and at www.cmaj.ca/lookup/doi/10.1503/cmaj.131650

$\mathrm{T}$ he World Health Organization classifies maternal deaths due to traffic crashes as coincidental and not related to the state of pregnancy. Others have argued that pregnancy is the root cause of such deaths, because pregnant women are more susceptible to crashes. In a linked $C M A J$ article, Redelmeier and colleagues ${ }^{1}$ report intriguing findings from a Canadian population-based study showing an excess risk of serious motor vehicle crashes during the second trimester of pregnancy compared with the risk before pregnancy. The study design is novel because the researchers used data from before pregnancy to use women as their own controls. The study also makes clever use of medical billing data routinely collected in Ontario with linkage to registries that supply data on sociodemographic characteristics and to the Canadian Community Health Survey for data on lifestyle factors. These robust methods address many of the flaws in previous research on the same topic. However, as with all observational studies, it is difficult to make causal inferences. In this article, we assess the associations shown in the study in light of the criteria proposed by Hill: ${ }^{2}$ temporality, consistency, biological plausibility and evidence of a dose-response effect.

Redelmeier and colleagues satisfy Hill's temporality criteria because the exposure (pregnancy) precedes the outcome (serious motor vehicle crash). Moreover, the study's findings are consistent with the findings of previous research. A population-based study from North Carolina found that the highest risk of a motor vehicle crash during pregnancy occurred at 2031 weeks' gestation, with a marked decline in the risk of a crash thereafter, ${ }^{3}$ which is similar to the current study's results. A decline in the risk of a motor vehicle crash among women in their last trimester of pregnancy could be explained by reduced numbers of women driving during the third trimester. A population-based casecontrol study that examined the association between birth defects and crashes among pregnant women also found that crashes were highest during the second trimester of a pregnancy. ${ }^{4}$

Examination of biological plausibility requires a discussion of the effects of normal physiologic adaptations of pregnancy that allow for the growth and survival of the fetus and ensure survival of the mother. Driving requires a high level of concentration and cognitive ability to maintain and complete a number of complex tasks. If there is any impairment in the driver's cognitive ability, there may be an increased risk of a crash. ${ }^{5}$ The physiologic changes of pregnancy have been shown to increase fatigue and sleep deprivation in pregnant women. ${ }^{6} \mathrm{~A}$ prospective study using self-reported questionnaires showed that sleep length began to decrease during the second trimester and quality of sleep worsened during pregnancy. ${ }^{7}$ Maternal stress is also a common feature of pregnancy. Exposure to everyday stressors through individual, societal and familial factors may encourage a physiologic response triggering negative maternal and perinatal outcomes in pregnant women. ${ }^{8} \mathrm{~A}$ review highlighted that drivers who experience sleep deprivation, stress or fatigue will have an increased risk of a car crash. ${ }^{5}$ Therefore, it is entirely feasible that an association between pregnancy and increased risk of a motor vehicle crash is facilitated by fatigue, sleep deprivation and maternal stress, which are normal characteristics of pregnancy. Redelmeier and colleagues showed a higher risk of motor vehicle crashes among women living in urban areas than among
Competing interests: None declared.

This article was solicited and has not been peer reviewed.

Correspondence to: Stephen J. McCall, smccall@abdn.ac.uk

CMAJ 2014. DOI:10.1503 /cmaj.140550 
those living in rural areas. If busy urban areas are harder to navigate and require greater concentration in driving, then fatigue, tiredness and stress are likely to have a greater impact on the risk of a crash in urban areas. ${ }^{9}$

If a dose-response effect existed, one might expect the rate of serious motor vehicle crashes to increase with increasing gestation of pregnancy. However, Redelmeier and colleagues show a specific association of increased risk of crash during the second trimester. One prospective study showed that pregnant women had similar rates of self-reported tiredness during early gestation and other periods in pregnancy. ${ }^{10} \mathrm{How}$ ever, another prospective study showed that fatigue, difficulty sleeping and headaches increased during the second trimester. ${ }^{6}$

There are a number of limitations to the current study that suggest the findings should be interpreted with caution. No data were available on the distance travelled and the frequency of journeys taken during the second trimester in comparison with baseline data. An important focus for further research would be the use of data on the amount of time a person drives to quantify the risk of a crash. ${ }^{3}$ The current study was also unable to distinguish the shared responsibility of traffic crashes, which is a major bias in all traffic studies. This is a particularly important point because Redelmeier and colleagues showed an increase in the relative risk of having a crash if multiple vehicles were involved. Furthermore, the primary outcome of a serious motor vehicle crash was measured by attendance at emergency services. Yet it is plausible that women who are in the second trimester of pregnancy are somewhat more cautious than they were before their pregnancy, and may be more likely to attend the emergency department even if not badly injured. Motor vehicle crashes at baseline may have been underreported given that worry about the well-being of an unborn baby was not an issue before pregnancy. ${ }^{11}$ This sugges- tion is borne out by the relative reduction in the risk of hospital admissions for motor vehicle crashes among pregnant women observed in the current study.

The results of Redelmeier and colleagues' study are consistent with previous research findings, and there is certainly biological plausibility supporting the notion of a causal link between pregnancy and high risk of a motor vehicle crash. However, the reported data cannot rule out the real possibility that women are more likely to seek medical help when injured during pregnancy.

\section{References}

1. Redelmeier DA, May SC, Thiruchlvam D, et al. Pregnancy and the risk of traffic crash. CMAJ 2014;186:742-50.

2. Hill AB. The environment and disease: Association or causation? Proc R Soc Med 1965;58:295-300.

3. Vladutiu CJ, Poole C, Marshall SW, et al. Pregnant driverassociated motor vehicle crashes in North Carolina, 2001-2008. Accid Anal Prev 2013;55:165-71.

4. Tinker SC, Reefhuis J, Dellinger AM, et al. Epidemiology of maternal injuries during pregnancy in a population-based study, 1997-2005. J Womens Health (Larchmt) 2010;19:2211-8.

5. Taylor AH, Dorn L. Stress, fatigue, health, and risk of road traffic crashes among professional drivers: the contribution of physical inactivity. Annu Rev Public Health 2006;27:371-91.

6. Rodriguez A, Bohlin G, Lindmark G. Symptoms across pregnancy in relation to psychosocial and biomedical factors. Acta Obstet Gynecol Scand 2001;80:213-23.

7. Hedman C, Pohjasvaara T, Tolonen U, et al. Effects of pregnancy on mothers' sleep. Sleep Med 2002;3:37-42.

8. Christian LM. Physiological reactivity to psychological stress in human pregnancy: current knowledge and future directions. Prog Neurobiol 2012;99:106-16.

9. Eiksund S. A geographical perspective on driving attitudes and behaviour among young adults in urban and rural Norway. Saf Sci 2009; 47:529-36

10. Okun ML, Kline CE, Roberts JM, et al. Prevalence of sleep deficiency in early gestation and its associations with stress and depressive symptoms. J Womens Health (Larchmt) 2013;22: 1028-37.

11. Weiss HB, Strotmeyer S. Characteristics of pregnant women in motor vehicle crashes. Inj Prev 2002;8:207-10.

Affiliations: Dugald Baird Centre for Research on Women's Health, Department of Obstetrics and Gynaecology, University of Aberdeen, Aberdeen, UK

Contributors: Stephen McCall drafted the manuscript, which Sohinee Bhattacharya revised. Both authors approved the final version submitted for publication and will act as guarantors for the work. 